\title{
Defect free optimization of a polycentric prosthetic knee design using imperialist competition-inspired optimization method
}

DOI:10.36909/jer.13063

\author{
M. Nejlaoui*, A. I. Alateyah*, W. H. El-Garaihy**** \\ * Department of Mechanical Engineering, College of Engineering, Qassim University, Unaizah, 51911, \\ Saudi Arabia \\ ** Mechanical Engineering Department, Faculty of Engineering, Suez Canal University, Ismailia 41522, \\ Egypt \\ Email: m.nejlaoui@qu.edu.sa; Corresponding Author.
}

\begin{abstract}
The disability of lower limbs is one of the major problems facing human lives. In order to restore the missing functionality and aesthetic feature of the amputee's locomotion, finding the optimal design of the human lower limb knee prosthesis is crucial. This paper focuses the design optimization of a four bar knee mechanism capable to reproduce the complex flexion/extension knee joint motion in the sagittal plane with variable instantaneous center of rotation positions. Thus an optimization approach with appropriate constraints is formulated in order to consider the degree of compatibility between the instantaneous center of rotation trajectories of the human reference knee joint and the four bar knee mechanism. To solve this high nonlinear optimization problem, an algorithm based on multi-objective modified imperialist competitive method is proposed where all the constraints are managed with the penalty method. The results obtained by the multi-objective modified imperialist competitive method showed the capability to reach a small tracking error. The obtained results prove the effectiveness of the proposed optimization approach for the optimal synthesis of the four bar knee mechanism, compared with other literature techniques.
\end{abstract}


Key words: four bar knee joint, defect free optimization, tracking error, imperialist competition method.

\section{INTRODUCTION}

For human beings, walking is an important everyday task. Several peoples have walking disabilities due to certain neuromuscular diseases, stroke, trauma, ageing..., which causes a lot of inconveniences in their daily lives. The most reliable way to restore the ability to walk is usually to use polycentric prostheses. The polycentric prosthetic usually contains FBKM, which is functionally flexible, topologically simple and easy in fabrication (Mohamed et al. 2017). A polycentric prosthetic knee should mimic the human knee motion. In other word, the instantaneous center of rotation (ICR) of the four bar knee mechanism (FBKM) should be very close to the ICR of the reference knee joint (Khan et al. 2015). Therefore, the dimensional optimization of FBKM to fulfill the reference movement is a challenge. Several determinist optimization methods are suggested (Krishnanand and Ghose 2009, Zhang, Zhou and Ye 2000). However, it is showed that the determinist method cannot converge to the global optimum, especially for constrained non-linear problems (Hosseini and Al Khaled 2014). To overcome these issues, stochastic methods can be used to optimize the FBKM. Genetic algorithms (GA) have been used to mechanism optimal synthesis (Khorshidi et al. 2011). Moreover, the particle swarm optimization (PSO) has been used in mechanisms synthesis (Eqra, Abiri and Vatankhah 2018, Syed et al. 2021). Differential evolution (DE) and simulated annealing (SA) have been used in the design optimization of four bar linkage with and without clearances (CHEN and ZHU 2009, Lin 2010, Naresh 2019). The imperialist competitive algorithm (ICA) has been also used to find the optimal design planar mechanisms (Bilel et al. 2018). Several researchers focused on studying the drawbacks of the above algorithms and developed other improved versions. For example, NSGAII, MOPSO, MGBICA are improved version of GA, PSO and ICA, respectively. 
In this context, MOMICA has recently been proposed as an efficient stochastic method that offers a best balance between convergence speed and diversity of solutions (Mohamed, Bilel and Alsagri 2020). Despite that, the MOMICA has not been

used in the prosthetic knee design because it is a quite recently tool.

In the optimization of FBKM, most researchers have not paid close attention to constraints formulations, despite their important role in finding defect free design (Bapat and Sujatha 2017, Bertomeu et al. 2007, Ghaemi et al. 2012, Kittisares et al. 2020, Muñoz-César et al. 2013). In reality, branch, Grashof and order defects often occur during the FBKM kinematic synthesis. If all possible orientations cannot be realized without dismantling the mechanism, a FBKM has a branch defect (Zhou and Cheung 2004). Grashof defect arises when the FBKM linkage needs to be fully rotated frequently, while order defect occurs when the FBKM linkage rotation is not in the desired order (Bilel et al. 2017). These defects are not avoided in literature works, except the Grashof one considered by a few researchers (Aoustin and Hamon 2013, Sancibrian et al. 2016). In this paper, the adequate constraints for Grashof, branch and order defects eliminations has been clearly established in the optimization problem of the FBKM. The objective function of this optimization problem is to minimize the error between the reference human knee ICR and the ICR of FBKM. For the first time, the design optimization of the prosthesis knee is conducted through the MOMICA developed quite recently in literature. The findings indicate the efficacy of the proposed optimization strategy compared with literature approaches.

\section{BIOMECHANICS OF HUMAN KNEE JOINT}

The human knee joint involves the femur's articulation over the tibia. The knee's primary role is to allow the joint rotation while absorbing and transmitting the load (Xu et al. 2016). The motion of the knee is generally modeled as a four-bar mechanism ABCD, as seen in Figure 1 (Dathe et al. 2016). The ICR is positioned at the point at which the anterior link AB and the posterior link 
DC cross. For different knee flexion angle, the ICR motion describes a trajectory.

Figure 2 illustrates some familiar prosthesis knee designs. To create a moving ICR as occurs with the human knee, the prosthesis knee usually includes four bar mechanisms where their interior and posterior links intersect in their ICR.

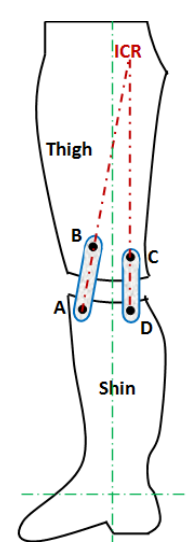

Figure 1 Four bar linkage as an equivalent of the human knee joint
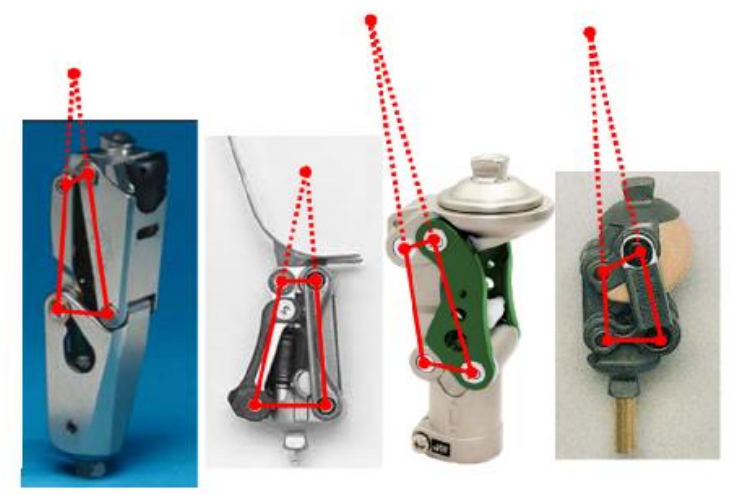

Figure 2 ICR of FBKM used in polycentric prosthesis (Etoundi, Vaidyanathan and Burgess 2012)

Ideally these ICR points should mimic the human knee ICR trajectory. Therefore, the FBKM configuration requires an optimization in order to follow a desired path.

\section{DETERMINATION OF ICR COORDINATES OF THE FBKM}

Figure 3 illustrates the adopted notations of the FBKM. The frame linkage (link 1) is positioned at the shank of the prosthesis. The coupler (link 3) is situated in the socket. The links 2 and 4 are positioned at both trimmings of link $1, \mathrm{~A}$ and $\mathrm{D}$. The position of $\mathrm{C}$ is given by:

$X_{C}=X_{D}+r_{4} \cos \theta_{4}=X_{A}+r_{2} \cos \theta_{2}+r_{3} \cos \theta_{3}$ 
$Y_{C}=Y_{D}+r_{4} \sin \theta_{4}=Y_{A}+r_{2} \sin \theta_{2}+r_{3} \sin \theta_{3}$

Equation (1) and (2) can be presented with respect to $\theta_{2}$, as:

$$
\begin{aligned}
& r_{2} \cos \theta_{2}=r_{4} \cos \theta_{4}+C_{1} \\
& r_{2} \sin \theta_{2}=r_{4} \sin \theta_{4}+C_{2}
\end{aligned}
$$

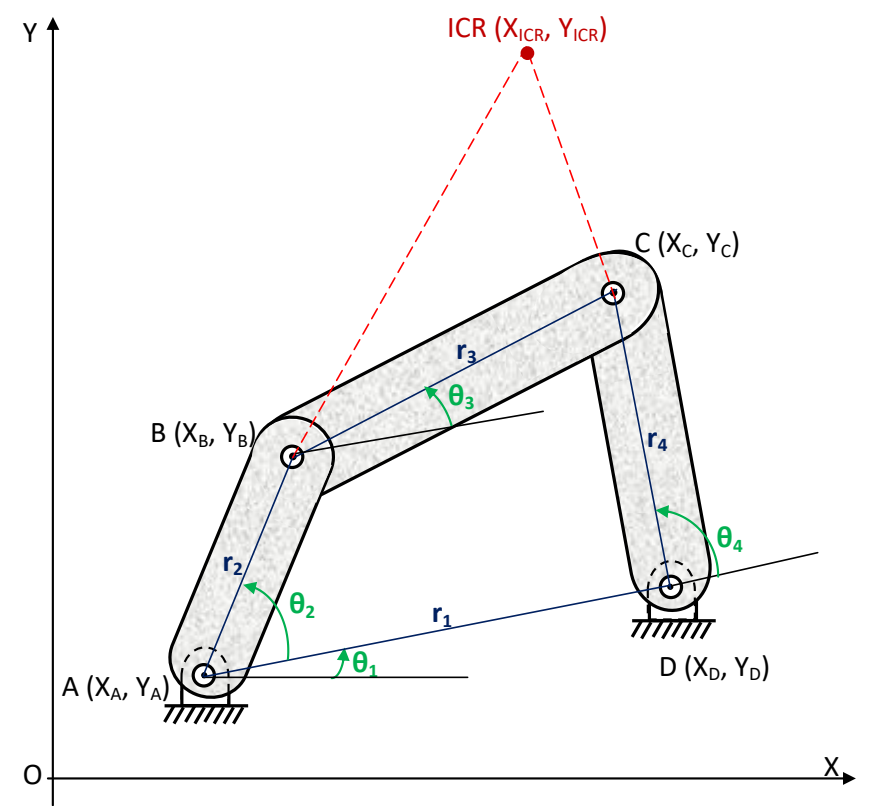

Figure 3 The FBKM configuration and adopted notations

Where

$C_{1}=X_{D}-X_{A}-r_{3} \cos \theta_{3}$

$C_{2}=Y_{D}-Y_{A}-r_{3} \sin \theta_{3}$

By squaring and adding equations (3) and (4), we obtain:

$r_{2}^{2}=r_{4}^{2}+C_{1}^{2}+2 C_{1} r_{4} \cos \theta_{4}+2 C_{2} r_{4} \sin \theta_{4}+C_{2}^{2}$

By rearranging Equation (7), we can obtain the following equation of motion:

$$
\begin{aligned}
& B \cos \theta_{4}+A \sin \theta_{4}=C \\
& A=2 C_{2} r_{4} \\
& B=2 C_{1} r_{4} \\
& C=r_{2}^{2}-r_{4}^{2}-C_{1}^{2}-C_{2}^{2}
\end{aligned}
$$


Equation (8) is a $\theta_{4}$ function. The following substitutions can make it explicit.

$$
\sin \theta_{4}=\frac{2 \tan \left(\frac{\theta_{4}}{2}\right)}{1+\tan ^{2}\left(\frac{\theta_{4}}{2}\right)}
$$

$$
\cos \theta_{4}=\frac{1-\tan ^{2}\left(\frac{\theta_{4}}{2}\right)}{1+\tan ^{2}\left(\frac{\theta_{4}}{2}\right)}
$$

By incorporating Equations (12) and (13) in (8), the latter is reduced in $\left(\frac{\theta_{4}}{2}\right)$ quadratic form. Its solution is given by the following equation (Muñoz-César et al. 2013):

$$
\theta_{4}=2 \tan ^{-1} \frac{A \pm \sqrt{A^{2}+B^{2}-C^{2}}}{B+C}
$$

As given in Equation (14), $\theta_{4}$ presents two solutions according to mechanism configuration.

The ICR of the FBKM can be calculated from the coordinates of the centers of rotation of link 2 and 4 and their angles (Muñoz-César et al. 2013):

$$
\begin{aligned}
& X_{I C R}=\frac{Y_{D}-Y_{A}-X_{D} \tan \theta_{4}-X_{A} \tan \theta_{2}}{\tan \theta_{2}-\tan \theta_{4}} \\
& Y_{I C R}=Y_{D}+\tan \theta_{4}\left(X_{I C R}-X_{D}\right)
\end{aligned}
$$

Equations (15) and (16) give the coordinates of any point on the ICR trajectory.

\section{PROCEDURE OF OPTIMIZATION}

\section{The optimization problem}

The purpose of the optimization problem is to find a FBKM design that mimics as closely as possible the Human knee movement (based on a Human knee ICR) while meeting the defined constraints. Thus, the objective function can be presented as a tracking error function (TE). 
$T E$ is defined by the sum of the Euclidien distance between the ICR trajectory $\left(X_{I C R}, Y_{I C R}\right)$ of FBKM and the reference human knee ICR trajectory $\left(X_{R / I C R}, Y_{R / I C R}\right)$ :

$$
T E(\mathbf{x})=\sum_{i=1}^{N} \sqrt{\left(X_{R / I C R}^{i}-X_{I C R}^{i}\right)^{2}+\left(Y_{R / I C R}^{i}-Y_{I C R}^{i}\right)^{2}}
$$

$N$ is the number of reference human knee ICR trajectory points.

$\mathbf{x}$ represents the vector of unknown design parameters, given by:

$$
\mathbf{x}=\left[r_{1}, r_{2}, r_{3}, r_{4}, X_{A}, Y_{A}, \theta_{1}, \theta_{2}^{1}, \theta_{2}^{2}, \theta_{2}^{3} \ldots ., \theta_{2}^{N}\right]
$$

The Grashof defect can be eliminated by satisfying the following Grashof rule.

$$
h_{1}(\mathbf{x})=r_{1}+r_{2}-r_{3}-r_{4}<0 \text { if }\left(r_{2}<r_{3}<r_{4}<r_{1}\right)
$$

The branch defect can be eliminated by considering the following constraint.

$$
h_{2}(\mathbf{x})=\theta_{3}^{i}-\theta_{4}^{i}<0 i=1, \ldots ., N
$$

Order defect occurs when the FBKM linkage rotation is not in the desired order (clockwise or counter-clockwise). To eliminate this defect, the following constraint is required.

$$
h_{3}(\mathbf{x})=\theta_{2}^{i}-\theta_{2}^{i+1}<0 i=1, \ldots, N
$$

The optimization problem regroups the objective function and the following constraints:

$$
\begin{array}{cl}
\text { Minimize: } & T E(\mathbf{x})=\sum_{i=1}^{N} \sqrt{\left(X_{R / I C R}^{i}-X_{I C R}^{i}\right)^{2}+\left(Y_{R / I C R}^{i}-Y_{I C R}^{i}\right)^{2}} \\
\text { Constraints: } & h_{1}(\mathbf{x})=r_{1}+r_{2}-r_{3}-r_{4}<0 \text { if }\left(r_{2}<r_{3}<r_{4}<r_{1}\right) \\
& h_{2}(\mathbf{x})=\theta_{3}^{i}-\theta_{4}^{i}<0 i=1, \ldots, N \\
& h_{3}(\mathbf{x})=\theta_{2}^{i}-\theta_{2}^{i+1}<0 i=1, \ldots, N \\
& L_{k} \leq x_{k} \leq U_{k} \quad k=1, \ldots ., n
\end{array}
$$

Where, the indices $k$ represents the design variables number. $L_{k}$ and $U_{k}$ define the lower and upper limits of the design variables search spaces, respectively.

\section{Optimization method}

The MOMICA method will be used to resolve the optimization problem. MOMICA has recently 
been proposed as an efficient stochastic method that offers a best balance between convergence speed and diversity of solutions (Mohamed et al. 2020). Figure 4 illustrates the different steps of MOMICA method. Firstly, the MOMICA starts a random generation of the initial population formed by many countries. The evaluation of different objective functions corresponding to each country yields to the determination of the countries costs. Based on this cost, some of powerful countries (with elevated costs) are considered as "imperialist" and the other countries are called “colonies". Each imperialist with some colonies form an empire. Thus, several initial empires are formed. Secondly, the assimilation step begins where colonies move towards their imperialists as illustrated in Figure 5-a (Mohamed et al. 2020).

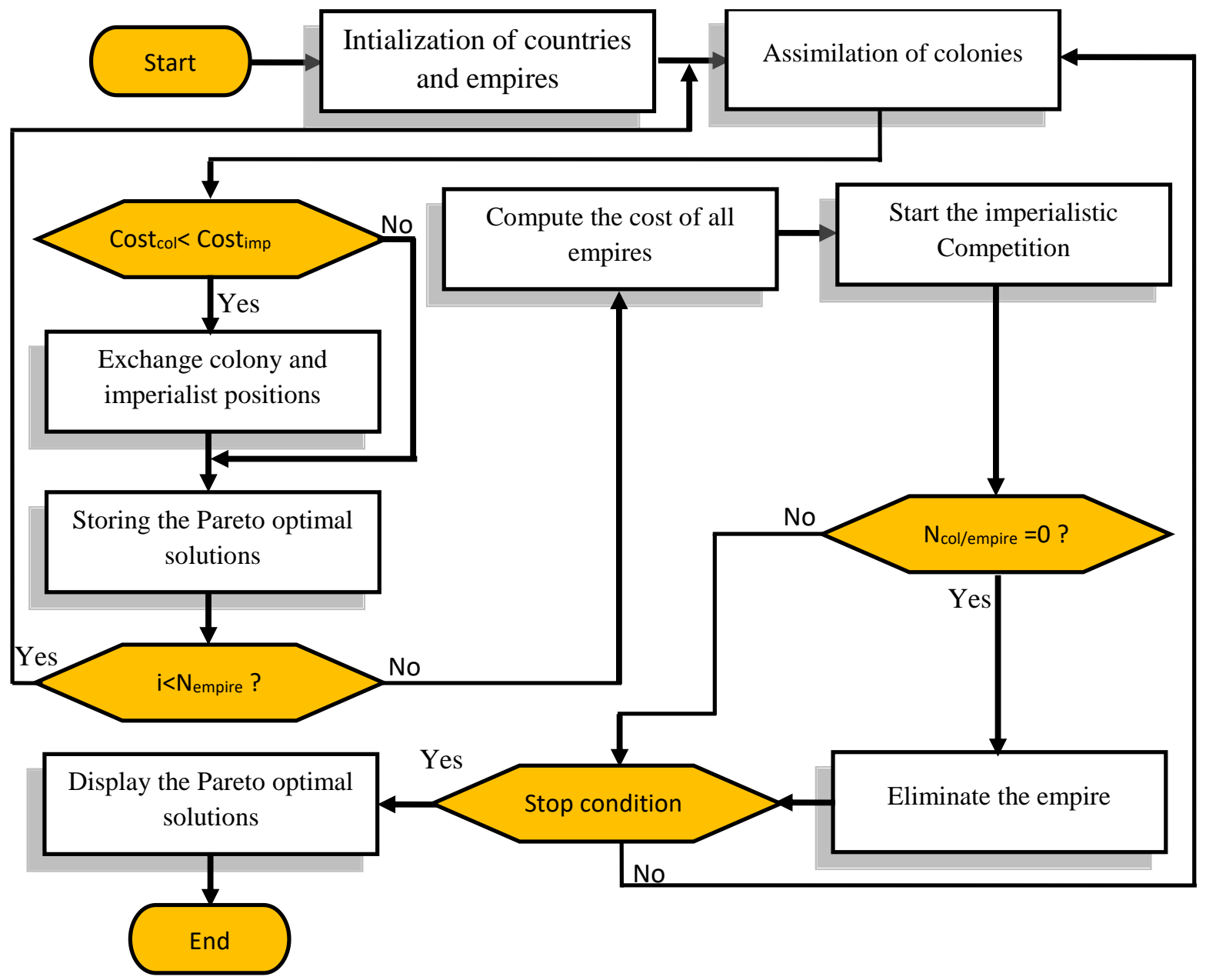

Figure 4 Different steps of MOMICA

After the assimilation phase, the costs of new colonies are recalculated (based on the objective 
function values) and compared with the cost of the imperialist. If any colony cost is less than the cost of its imperialist, then the imperialist should be swapped with that colony. Thirdly, imperialist competition occurs as presented in Figure 5-b. In fact, dominant empires acquire colonies of weaker ones (Mohamed et al. 2020). After several iterations, weaker empires will lose all their colonies and fall. This process repeats until remain only one empire.

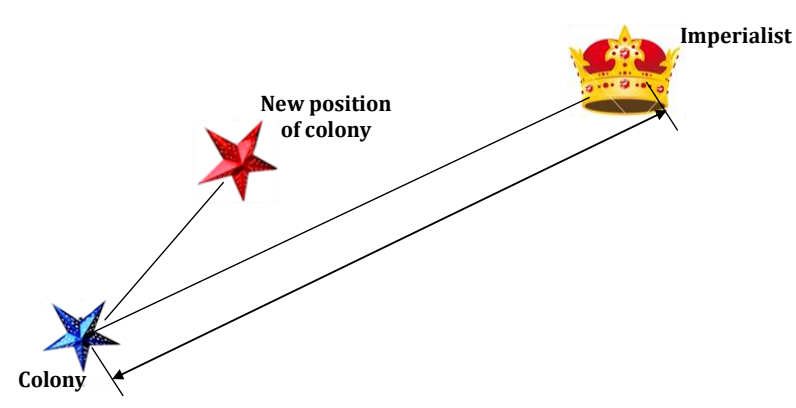

a)

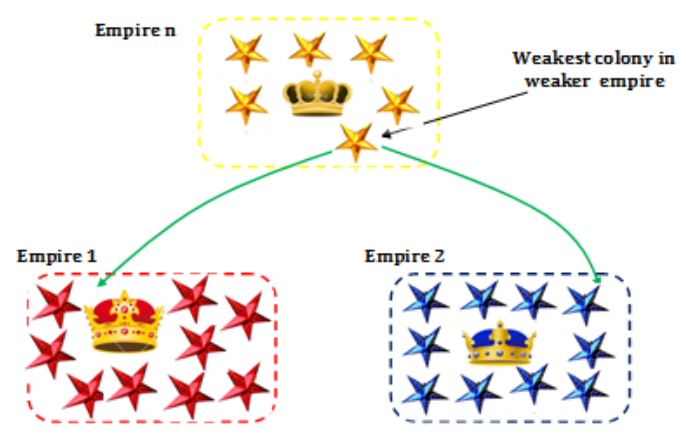

b)

Figure 5 Empires improvements; a) Colonies movement $\quad$ b) Competition stage

\section{RESULTS OF OPTIMIZATION}

The MOMICA method is applied for the optimization of the defect free FBKM for flexion/extension motion of human knee. The reference human knee ICR trajectory is illustrated in Table 1. The ranges of the different FBKM design parameters are illustrated in Table 2.

Table 1 Reference knee ICR trajectory (Sancibrian et al. 2016, Singh, Chaudhary and Singh 2017)

\begin{tabular}{|c|c|c|c|c|c|c|c|c|c|c|c|c|c|c|}
\hline Points & $\mathbf{1}$ & $\mathbf{2}$ & $\mathbf{3}$ & $\mathbf{4}$ & $\mathbf{5}$ & $\mathbf{6}$ & $\mathbf{7}$ & $\mathbf{8}$ & $\mathbf{9}$ & $\mathbf{1 0}$ & $\mathbf{1 1}$ & $\mathbf{1 2}$ & $\mathbf{1 3}$ & $\mathbf{1 4}$ \\
\hline $\mathrm{X}_{\mathrm{R} / \mathrm{ICR}}$ & -50 & -16 & 10 & 14 & 6 & 0 & -5 & -10 & -15 & -20 & -25 & -30 & -35 & -40 \\
\hline$Y_{R / I C R}$ & 200 & 150 & 100 & 60 & 18 & 0 & -12 & -19 & -21.5 & -22 & -21 & -18 & -11 & 0 \\
\hline
\end{tabular}

Table 2 Variables search domains (Sancibrian et al. 2016, Singh et al. 2017)

\begin{tabular}{|l|c|c|c|c|c|c|c|c|}
\hline $\mathbf{x}$ & $\mathrm{r}_{1}(\mathrm{~mm})$ & $\mathrm{r}_{2}(\mathrm{~mm})$ & $\mathrm{r}_{3}(\mathrm{~mm})$ & $\mathrm{r}_{4}(\mathrm{~mm})$ & $\mathrm{X}_{\mathrm{A}}(\mathrm{mm})$ & $\mathrm{Y}_{\mathrm{A}}(\mathrm{mm})$ & $\theta_{1}\left({ }^{\circ}\right)$ & $\theta_{2}^{\mathrm{i}}\left({ }^{\circ}\right)$ \\
\hline$L_{k}$ & 20 & 40 & 20 & 40 & -1500 & -1500 & 0 & 0 \\
\hline$U_{k}$ & 90 & 170 & 90 & 170 & 1500 & 1500 & 360 & 360 \\
\hline
\end{tabular}

To evaluate the MOMICA efficacy, the same optimization problem is solved by using the well- 
known natural inspired methods (GA and MOPSO). The evolution of $T E$ as function of the number of iteration, for the best FBKM design, is presented in Figure 6. One can note from Figure 6, that the lower value of $T E$ is given by MOMICA, compared with GA and MOPSO. In fact, GA reached an optimum value of $12 \mathrm{~mm}$ in 150 iterations, MOPSO offered $22 \mathrm{~mm}$ in 200 iterations and MOMICA provided $6.7 \mathrm{~mm}$ in 178 iterations. Thus, MOMICA is capable to improve the TE results of GA and MOPSO about $44 \%$ and $69 \%$, respectively.

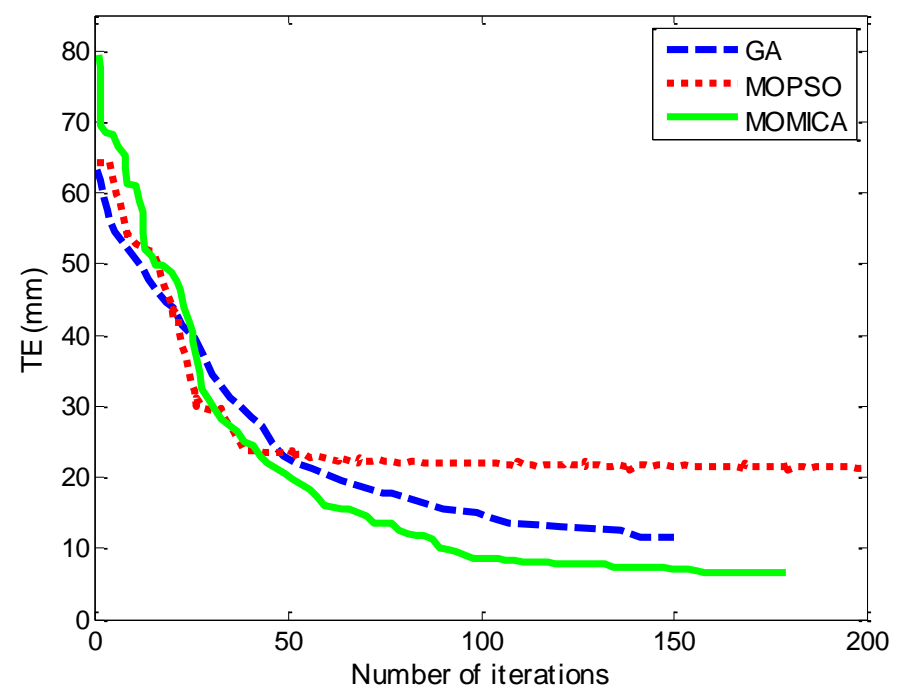

Figure 6 Convergence of $T E$ with GA, MOPSO and MOMICA

The design parameters of the optimal of FBKM are detailed in Table 3.

Table 3 Optimum FBKM obtained by GA, MOPSO and MOMICA

\begin{tabular}{|l|c|c|c|c|c|c|c|c|}
\hline $\mathbf{x}$ & $\mathrm{r}_{1}(\mathrm{~mm})$ & $\mathrm{r}_{2}(\mathrm{~mm})$ & $\mathrm{r}_{3}(\mathrm{~mm})$ & $\mathrm{r}_{4}(\mathrm{~mm})$ & $\mathrm{X}_{\mathrm{A}}(\mathrm{mm})$ & $\mathrm{Y}_{\mathrm{A}}(\mathrm{mm})$ & $\theta_{1}\left({ }^{\circ}\right)$ & $T E(\mathrm{~mm})$ \\
\hline GA & 88.43 & 52.13 & 65.16 & 79.77 & -101.86 & -313.22 & 28.56 & 12 \\
\hline MOPSO & 88.76 & 54.54 & 65.09 & 81.21 & -219.33 & -278.98 & 42.66 & 22 \\
\hline MOMICA & 79.92 & 55.05 & 65.72 & 74.54 & -373.44 & -354.88 & 17.39 & $\mathbf{6 . 7}$ \\
\hline
\end{tabular}

Figure 7 demonstrates the generated trajectories by GA, MOPSO and MOMICA. The reference human knee ICR trajectory is also presented in the same figure. 


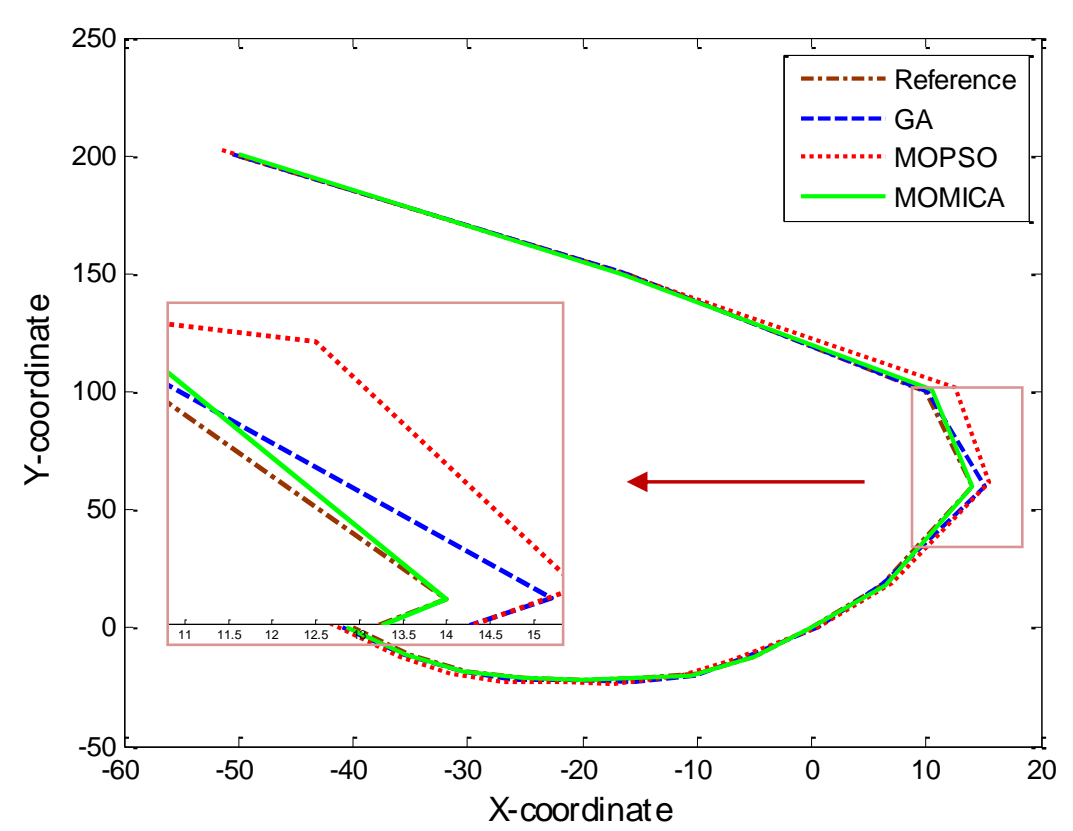

Figure 7 Different ICR paths (Reference, GA, MOPSO, MOMICA)

It is shown that the MOMICA trajectory is the closest to the reference ICR path, compared with GA and MOPSO. The errors between each reference point and the ICR trajectories are presented in Figure 8. It is noted that MOMICA dominates GA and MOPSO in all reference points, except in point 3. This proves the efficacy of MOMICA in the FBKM design optimization.

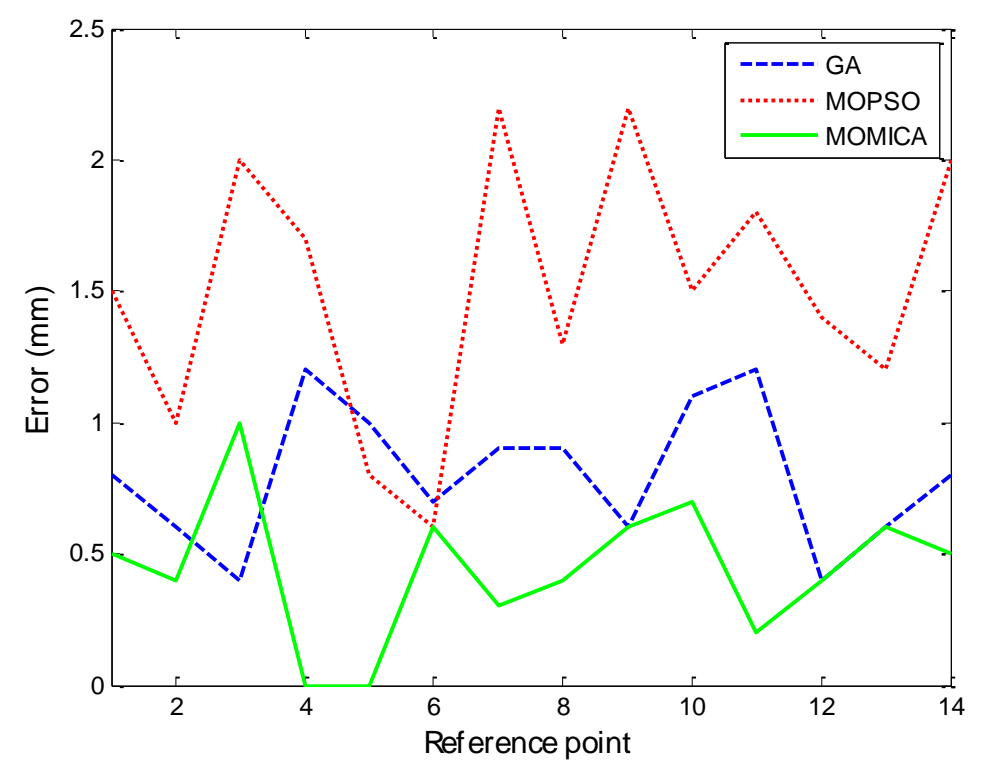

Figure 8 Evolution of errors as function of reference points

Table 4 resumes the minimum, average and maximum errors to the reference human knee ICR path, obtained by different optimization methods. One can note that MOMICA results in terms of 
minimum, average and maximum errors are lower than those given by GA, MOPSO, TLBO and NSGAII. This result proves the effectiveness of MOMICA method especially in the FBKM optimization. In fact, in MOMICA, an attraction and repulsion concept is implemented in the assimilation phase to improve the performances of the algorithm to reach the global optimal position and ameliorate the diversity rates of the algorithm (Mohamed et al. 2020)

Table 4 Optimum FBKM obtained by GA, MOPSO and MOMICA

\begin{tabular}{|c|c|c|c|c|c|}
\hline Error & GA & MOPSO & MOMICA & $\begin{array}{c}\text { TLBO (Singh et } \\
\text { al. 2017) }\end{array}$ & $\begin{array}{c}\text { NSGA II (Bertomeu } \\
\text { et al. 2007) }\end{array}$ \\
\hline Minimum & 0.43 & 0.62 & $\mathbf{0 . 0 0 1}$ & 0.86 & 0.63 \\
\hline Average & 0.81 & 1.57 & $\mathbf{0 . 4 1}$ & 3.2 & 1.27 \\
\hline Maximum & 1.28 & 2.21 & $\mathbf{1 . 0 5}$ & 9.21 & 1.91 \\
\hline
\end{tabular}

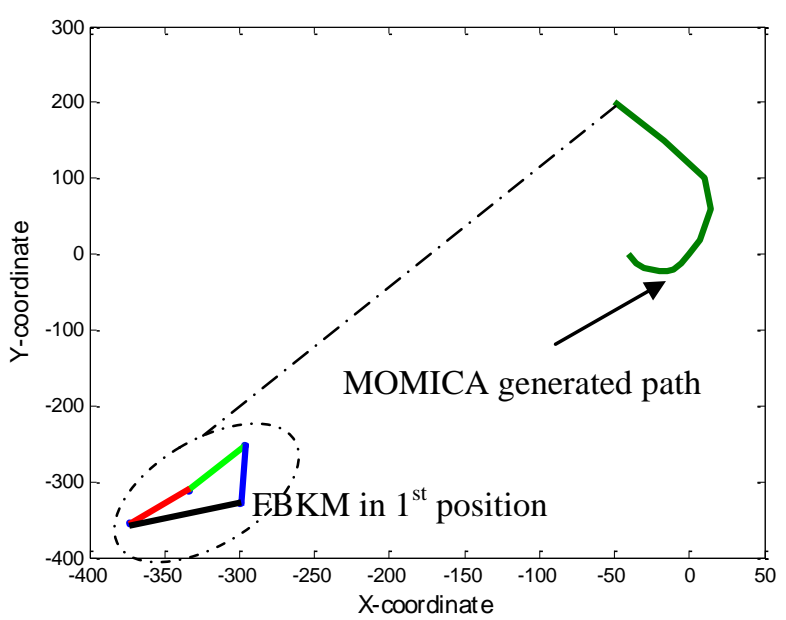

(a)

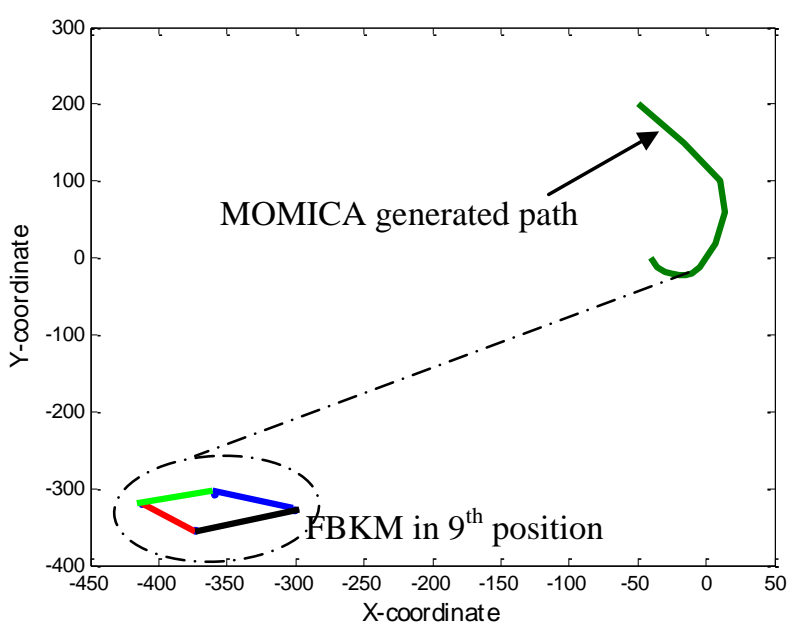

(c)

Figure 9 FBKM motion Simulation: a) $1^{\text {st }}$ position

c) $9^{\text {th }}$ position

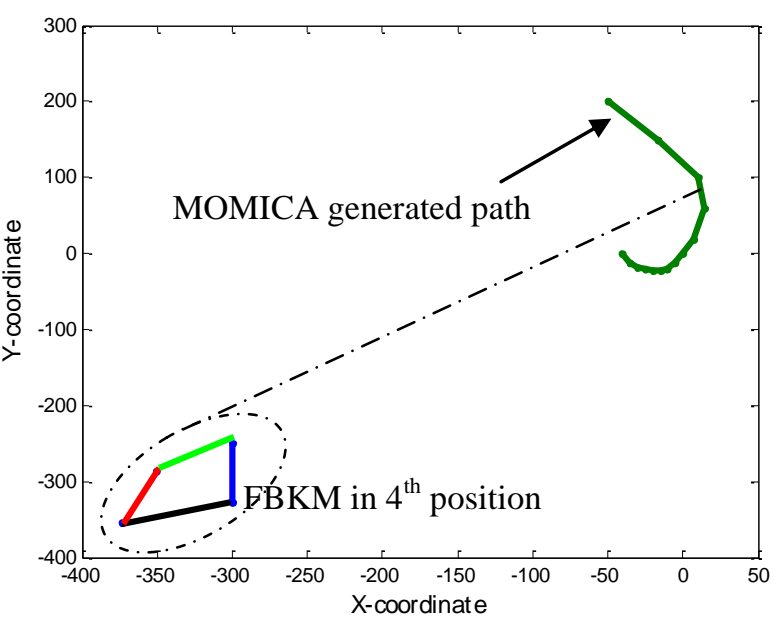

(b)

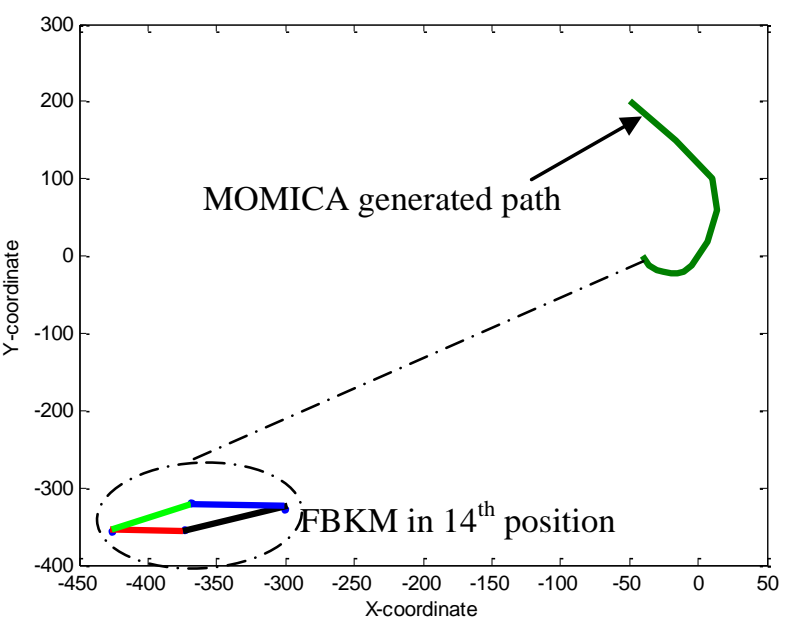

(d)

d) $14^{\text {th }}$ position 
In order to verify that the developed FBKM is a defect free system, it is necessary to check the Grashof, branch and order constraints adopted in the optimization strategy. Figure 9 presents four sequential positions of the FBKM corresponding to four randomly selected values of $\theta_{2}$. This confirms that the system travels sequentially without any stationary configuration which proves that the Grashof defect is avoided. Figure 10-a represents the $\theta_{2}$ evolution as function of the 14 sequential reference points. One can note that by passing from one reference point to the successive one, the value of $\theta_{2}$ increases. Therefore, the link 2 rotation is in the counterclockwise order. Hence the order defect is eliminated. Figure 10-b demonstrates the evolution of $\theta_{3}-\theta_{4}$ values as function of the 14 reference points. One can note that for all the reference points, $\theta_{3}$ always inferior to $\theta_{4}$. Therefore, the branch defect is avoided.

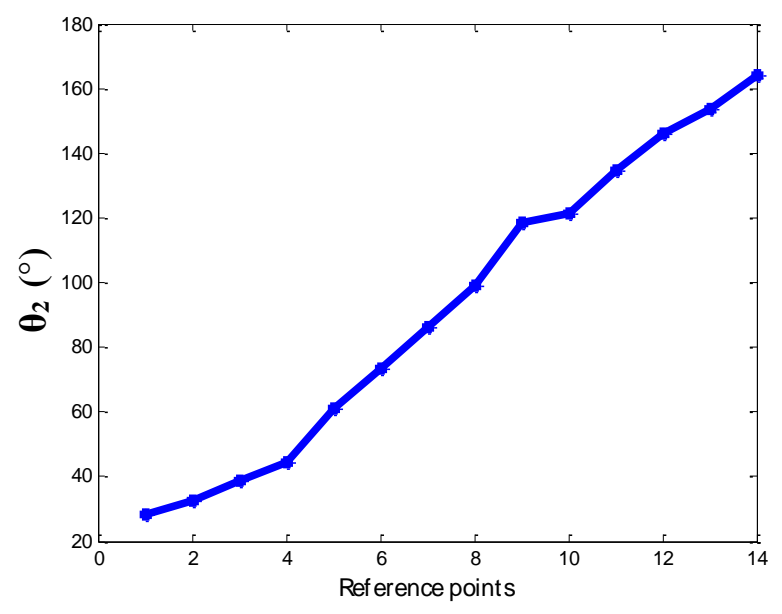

a)

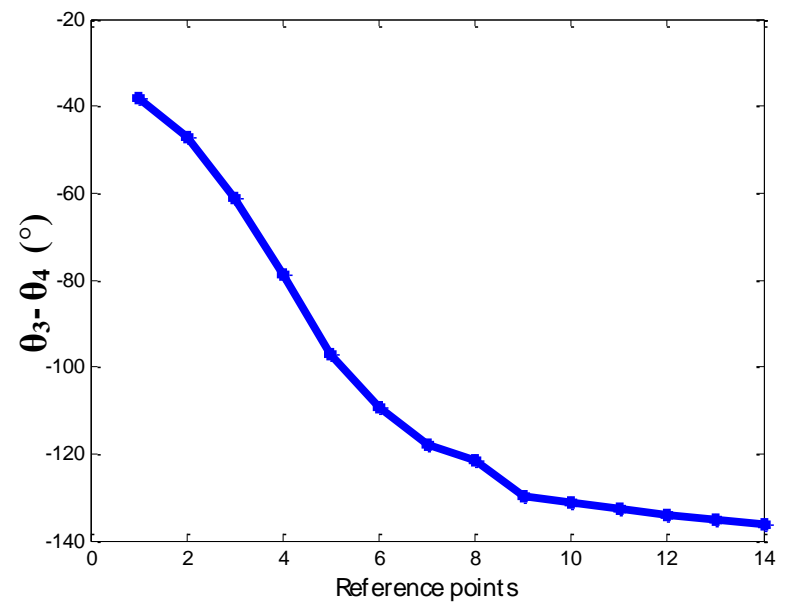

b)

Figure 10 Angle evolution as function of reference points: a) $\theta_{2}$ evolution b) $\theta_{3}-\theta_{4}$ evolution

The presented results turn out to be a valuable tool for the designer to choose the defect free optimal FBKM. In fact, the proposed optimization approach could help the designer in the choice of the most adequate design parameters yielding the FBKM with the minimum tracking error. 


\section{CONCLUSION}

This paper presented a new design of the four bar knee mechanism by adopting the appropriate constraints that avoid the Grashof, branch and order defects. An optimization strategy based on the multi-objective modified imperialist competitive algorithm (MOMICA) is developed to solve this nonlinear problem. For comparison reasons, GA and MOPSO are also used to solve the optimization problem. It is showed that the MOMICA can offer a FBKM tracking error improved about $44 \%$ and $69 \%$ than those given by GA and MOPSO, respectively. In addition, it is founded that the proposed MOMICA mechanisms satisfy all the constraints used for the defect free optimization and without any stationary configuration. Thus, the proposed optimization strategy by using MOMICA, proves to effective tool for the optimal synthesis of the FBKM.

\section{References}

Aoustin, Y. \& A. Hamon. 2013. Human like trajectory generation for a biped robot with a fourbar linkage for the knees. Robotics and Autonomous Systems, 61, 1717-1725.

Bapat, G. M. \& S. Sujatha. 2017. A method for optimal synthesis of a biomimetic four-bar linkage knee joint for a knee-ankle-foot orthosis. In Journal of Biomimetics, Biomaterials and Biomedical Engineering, 20-28. Trans Tech Publ.

Bertomeu, J. M. B., J. M. B. Lois, R. B. Guillem, Á. P. Del Pozo, J. Lacuesta, C. G. Mollà, P. V. Luna \& J. P. Pastor. 2007. Development of a hinge compatible with the kinematics of the knee joint. Prosthetics and orthotics international, 31, 371-383.

Bilel, N., N. Mohamed, A. Zouhaier \& R. Lotfi .2017. Multi-objective robust design optimization of a mechatronic system with uncertain parameters, using a polynomial chaos expansion method. Proceedings of the Institution of Mechanical Engineers, Part I: Journal of Systems and Control Engineering, 231, 729-739.

Bilel, N., N. Mohamed, A. Zouhaier \& R. Lotfi. 2018. Mechatronic design optimization of the mechanism in a sewing machine. Proceedings of the Institution of Mechanical Engineers, Part C: Journal of Mechanical Engineering Science, 232, 542-556.

CHEN, X.-j. \& Y.-k. ZHU. 2009. Optimization Design of Four-bar Linkage Based on Genetic Simulated Annealing Algorithm. Mechanical Engineering \& Automation, 16.

Dathe, H., R. Gezzi, C. Fiedler, D. Kubein-Meesenburg \& H. Nägerl. 2016. The description of the human knee as four-bar linkage. Acta of bioengineering and biomechanics, 18.

Eqra, N., A. H. Abiri \& R. Vatankhah. 2018. Optimal synthesis of a four-bar linkage for path generation using adaptive PSO. Journal of the Brazilian Society of Mechanical Sciences and Engineering, 40, 469.

Etoundi, A., R. Vaidyanathan \& S. Burgess. 2012. A bio-inspired condylar knee joint for leg amputees and for knee implants. Design and Nature VI, 160, 23-34.

Ghaemi, N., M. Dardel, M. H. Ghasemi \& H. Zohoor. 2012. Optimization of six bar knee linkage for stability of knee prosthesis. Majlesi Journal of Mechatronic Systems, 1. 
Hosseini, S. \& A. Al Khaled. 2014. A survey on the imperialist competitive algorithm metaheuristic: implementation in engineering domain and directions for future research. Applied Soft Computing, 24, 1078-1094.

Khan, H., R. Featherstone, D. G. Caldwell \& C. Semini. 2015. Bio-inspired knee joint mechanism for a hydraulic quadruped robot. In 2015 6th International Conference on Automation, Robotics and Applications (ICARA), 325-331. IEEE.

Khorshidi, M., M. Soheilypour, M. Peyro, A. Atai \& M. S. Panahi. 2011. Optimal design of four-bar mechanisms using a hybrid multi-objective GA with adaptive local search. Mechanism and Machine Theory, 46, 1453-1465.

Kittisares, S., H. Nabae, G. Endo, K. Suzumori \& R. Sakurai. 2020. Design of knee support device based on four-bar linkage and hydraulic artificial muscle. ROBOMECH Journal, 7, $1-10$.

Krishnanand, K. \& D. Ghose. 2009. Glowworm swarm optimization for simultaneous capture of multiple local optima of multimodal functions. Swarm intelligence, 3, 87-124.

Lin, W.-Y. 2010. A GA-DE hybrid evolutionary algorithm for path synthesis of four-bar linkage. Mechanism and Machine Theory, 45, 1096-1107.

Mohamed, N., N. Bilel \& A. S. Alsagri. 2020. A multi-objective methodology for multi-criteria engineering design. Applied Soft Computing, 106204.

Mohamed, N., N. Bilel, A. Zouhaier \& R. Lotfi. 2017. Multi-objective design optimisation of four-bar mechanisms using a hybrid ICA-GA algorithm. International Journal of Reasoning-based Intelligent Systems, 9, 43-51.

Muñoz-César, J. J., L. H. Hernández-Gómez, O. I. López-Suárez, G. Urriolagoitia-Sosa, J. A. Beltrán-Fernández, G. Urriolagoitia-Calderón, N. D. Pava-Chipol \& I. J. Quintero-Gómez. 2013. Optimization of the design of a four bar mechanism for a lower limb prosthesis using the taboo search algorithm. In Advances in Bio-Mechanical Systems and Materials, 107-125. Springer.

Naresh, K., Y. 2019. Hybridization of Particle Swarm Optimization with Differential Evolution for Solving Combined Economic Emission Dispatch Model for Smart Grid. Journal of Engg. Research Vol. 7 No. (3) pp. 244-257

Sancibrian, R., E. G. Sarabia, A. Sedano \& J. M. Blanco. 2016. A general method for the optimal synthesis of mechanisms using prescribed instant center positions. Applied Mathematical Modelling, 40, 2206-2222.

Syed S., F., A. A. Baqai \& M. F. Shah. 2021. Optimal design of tricept parallel manipulator with particle swarm optimization using performance parameters. Journal of Engg. Research Vol. 9 No. (2) pp. 278-295

Singh, R., H. Chaudhary \& A. K. Singh. 2017. Defect-free optimal synthesis of crank-rocker linkage using nature-inspired optimization algorithms. Mechanism and Machine Theory, 116, 105-122.

Xu, L., D.-H. Wang, Q. Fu, G. Yuan \& L.-Z. Hu. 2016. A novel four-bar linkage prosthetic knee based on magnetorheological effect: principle, structure, simulation and control. Smart Materials and Structures, 25, 115007.

Zhang, X., J. Zhou \& Y. Ye. 2000. Optimal mechanism design using interior-point methods. Mechanism and Machine Theory, 35, 83-98.

Zhou, H. \& E. H. Cheung. 2004. Adjustable four-bar linkages for multi-phase motion generation. Mechanism and Machine Theory, 39, 261-279. 